en salud. (Regidor E. Social determinants of health: a veil that hides socioeconomic position and its relation with health. J Epidemiol Community Health. 2006;60:896-901.)

\section{Efecto independiente de la exposición pasiva prenatal y posnatal al tabaco}

La exposición pasiva al tabaco (EPT), tanto prenatal como posnatal, está asociada con las afecciones respiratorias y el asma durante la infancia. Sin embargo, se desconoce la contribución individual de cada una de estas formas de EPT a los trastornos pulmonares en la población infantil. En este artículo se analiza el efecto que tiene sobre la función respiratoria la EPT prenatal y posnatal de niños en edad escolar de varios países con características geográficas, sociales y culturales muy diferentes entre sí.

Se utilizaron los datos de volumen y flujo respiratorios de ocho estudios transversales que forman parte del estudio Polución y Juventud (PATY), que abarcó 57363 niños de 10 países europeos, Rusia y América del Norte. En total se analizaron 22712 niños, de los cuales se contaba con la información sobre la asociación independiente entre los indicadores de la función pulmonar y la EPT en la etapa prenatal (hábito de fumar de la madre durante el embarazo) y posnatal (durante los primeros 2 años de vida del niño y en el momento de la investigación). La información sobre la EPT se obtuvo mediante cuestionarios y la prueba de espirometría se realizó de manera estandarizada según el protocolo establecido por la Sociedad Estadounidense del Tórax y la Sociedad Europea de Enfermedades Respiratorias.

La frecuencia del hábito de fumar durante el embarazo varió entre 4,9\% en Alemania y 28,1\% en Holanda. Según la regresión logística se observó un aumento en el riesgo de disminución de la función pulmonar (31\% del flujo pico espiratorio y $40 \%$ del flujo medio espiratorio máximo) en los niños expuestos al tabaquismo de la madre durante el embarazo. En el modelo parcialmente ajustado, el efecto fue menor (15\% y 30\%, respectivamente), pero mantuvo su significación estadística.
A pesar de las diferencias geográficas, sociales y ambientales, se observó una gran coherencia entre los resultados de todos los estudios analizados. El hábito de fumar durante el embarazo tuvo un mayor efecto sobre los parámetros de flujo que en el volumen espirado durante el primer segundo de una espiración forzada.

El efecto de la EPT encontrado en este estudio afectó poco a la función pulmonar media de la población, pero contribuyó a aumentar el porcentaje de niños con actividad pulmonar deficiente. El efecto del hábito de fumar durante el embarazo y de la EPT actual se redujo después de ajustar por algunos posibles factores de confusión. Sin embargo, algunos de esos factores, como el hacinamiento o el bajo estatus socioeconómico (medido según el nivel educacional de los padres), pueden haber influido en la magnitud del efecto de la EPT por el efecto añadido de las infecciones respiratorias que pudieran haber sido favorecidas por la EPT.

Se encontró un mayor efecto negativo, aunque pequeño, de la EPT en niños que en niñas. El hábito de fumar afectó más a los niños que no padecían de asma que a los asmáticos, aunque esto pudo deberse al subdiagnóstico del asma en los niños de familias de menor estatus socioeconómico, en las que la EPT tiende a ser mayor. No se observó una clara tendencia relacionada con la edad, lo que resulta interesante, ya que esto hubiera reflejado el debilitamiento del efecto sufrido por haber estado sometido a EPT solamente durante el embarazo.

En conclusión, los resultados de este estudio confirman en una gran muestra multinacional el efecto negativo que provoca el hábito de fumar de las mujeres durante el embarazo sobre la actividad pulmonar posterior de sus hijos. Este efecto fue mayor que el daño causado por la EPT durante la infancia. Además, demuestran que ambos efectos son independientes. Tomando en cuenta el elevado número de niños expuestos, estos resultados indican que la EPT, tanto durante el embarazo como en la infancia, constituye un grave problema de salud pública. (Moshammer H, Hoek G, LuttmannGibson H, Neuberger MA, Antova T, Gehring U, et al. Parental smoking and lung function in children. An international study. Tob Control. 2006;15: 294-301.) 